\title{
On chi-square type distributions with geometric degrees of freedom in relation to geometric sums
}

\section{Tran Loc Hung*}

University of Finance and Marketing

\section{Correspondence}

Tran Loc Hung, University of Finance and Marketing

Email: tlhung@ufm.edu.vn

\section{History}

- Received: 2018-11-21

- Accepted: 2019-03-24

- Published: 2019-03-31

DOI :

https://doi.org/10.32508/stdj.v22i1.1053

\section{Check for updates}

\section{Copyright}

(c) VNU-HCM Press. This is an openaccess article distributed under the terms of the Creative Commons Attribution 4.0 International license.

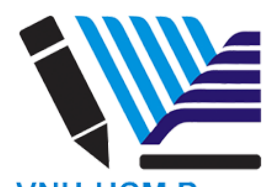

VNU-HCM Press

\begin{abstract}
The chi-square distribution with $n$ degrees of freedom has an important role in probability, statistics and various applied fields as a special probability distribution. This paper concerns the relations between geometric random sums and chi-square type distributions whose degrees of freedom are geometric random variables. Some characterizations of chi-square type random variables with geometric degrees of freedom are calculated. Moreover, several weak limit theorems for the sequences of chi-square type random variables with geometric random degrees of freedom are established via asymptotic behaviors of normalized geometric random sums.

MSC2010: Primary 60E05; 60E07; Secondary 60F05; 60G50.

Key words: Chi-square distribution, Geometric random sums, Weak limit theorems.
\end{abstract}

\section{INTRODUCTION}

Let $\left\{X_{n}, n \geq 1\right\}$ be a sequence of independent, standard normal distributed random variables, (shortly, $\left.X_{n} \sim \mathscr{N}(0,1), n \geq 1\right)$.

It has long been known that the partial sum $X_{1}^{2}+X_{2}^{2}+$ $\cdots+X_{n}^{2}$ is said to be a chi-square random variable with $n$ degrees of freedom, denoted by $\chi^{2}(n)$. The probability density function of the $\chi^{2}(n)$ is given by

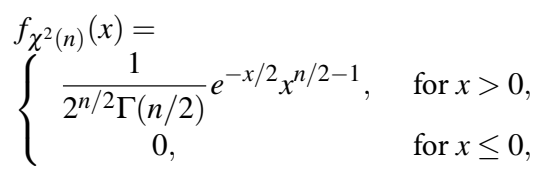

where $\Gamma(\mathrm{y})=\int_{0}^{+\infty} e^{-x} x^{y-1} d x$ (for $y>1$ ), denotes the Gamma function (see for instance ${ }^{1}$ ). It is easily seen that $\left\{X_{j}^{2}, j \geq 1\right\}$ is a sequence of independent, identically distributed (i.i.d.) random variables, $X_{j}^{2} \sim \chi^{2}(1)$ for $j \geq 1$ with mean $\mathbb{E}\left(X_{j}^{2}\right)=1$ and finite variance $\operatorname{Var}\left(X_{j}^{2}\right)=2$, for all $j \geq 1$.

Thus, the chi-square random variable $\chi^{2}(n)$ should be considered as a partial sum of $n$ desired i.i.d random variables $X_{j}^{2}, j \geq 1$. Especially, degree of freedom of chi-square distribution $\chi^{2}(n)$ is a deterministic number $n$ of square of i.i.d. random variables having standard normal distribution in summation.

It is also worth pointing out that for large $n$ the desired sequence $\left\{X_{j}^{2}, j \geq 1\right\}$ will be obeyed the classical weak limit theorems like weak law of large numbers and central limit theorem. Especially, the classi- cal Weak law of large numbers states that

$$
\begin{aligned}
& \frac{\chi^{2}(n)-\mathbb{E}\left(\chi^{2}(n)\right)}{n}= \\
& n^{-1} \sum_{j-1}^{n} X_{j}^{2} \stackrel{P}{\rightarrow} 1 \quad \text { as } n \rightarrow \infty .
\end{aligned}
$$

or

$$
n^{-1} \sum_{j=1}^{n} X_{j}^{2} \stackrel{D}{\rightarrow} \mathscr{D}_{1} \text { as } n \rightarrow \infty
$$

where $\mathscr{D}_{1}$ is a random variable degenerated at point 1. Furthermore, the Central limit theorem will be formulated as follow

$$
\begin{aligned}
& \frac{\chi^{2}(n)-\mathbb{E}\left(\chi^{2}(n)\right)}{\left[\operatorname{Var}\left(\chi^{2}(n)\right)\right]^{1 / 2}}= \\
& n^{-1 / 2} \sum_{j=1}^{n}\left(\frac{X_{j}^{2}-1}{\sqrt{2}}\right) \stackrel{D}{\rightarrow} \mathscr{N}(0,1) \text { as } n \rightarrow \infty .
\end{aligned}
$$

(see for instance ${ }^{1}$, page 156-159). Here and subsequently, the symbols $P$ and $\begin{aligned} & D \\ & \rightarrow\end{aligned}$ stand for the convergence in probability and convergence in distribution, respectively. The chi-square distribution with degrees of freedom $n$ plays an important role in various applied problems like $\chi^{2}-$ testing in nonparametric statistics, in estimation theory or in testing hypothesis, etc. (see ${ }^{1}$ for more details).

The interesting question arises as to what happens with the distribution of

chi-square random variable with $n$ degrees of freedom, when the deterministic number $n$ (degree of freedom) will be replaced by a positive-integer valued random variable $N$, which independent of all 
$X_{n}, n \geq 1$. This question has been addressed in the $\operatorname{article}^{2}$. Moreover, the results should be more interesting if the degree of freedom being a geometric random variable $N_{p}, p \in(0,1)$, independent of all $X_{j}, j \geq 1$ and having a probability mass function $P\left(N_{p}=k\right)=p(1-p)^{k-1}, k \geq 1, p \in(0,1)$. Then the sum $X_{1}^{2}+X_{2}^{2}+\cdots+X_{N_{p}}^{2}$ of random variables $X_{n}^{2}$ up to the geometric degrees of freedom, denoted by $\chi^{2}\left(N_{p}\right)$. On the other hand, the $\chi^{2}\left(N_{p}\right)$ may be considered in the role of a compound geometric sum $\chi^{2}\left(N_{p}\right):=X_{1}^{2}+X_{2}^{2}+\cdots+X_{N_{p}}^{2}$, which will lead to interesting results, too. Itshould be noted that in the classical literature the compound geometric sums have been attracting much attention. Actually, the compound geometric sums can model many phenomena in insurance, queuing, finances, reliability, biology, storage, and other real world fields (for a deeper discussion of this the reader is referred to 3456789 ).

This paper deals with study of the distribution of chisquare type random

variables with geometric degrees of freedom via geometric random sums. Some characterizations of the $\chi^{2}\left(N_{p}\right)$ are given. Two asymptotic results of the probability distribution functions of the $\chi^{2}\left(N_{p}\right)$ are also investigated in two limit theorems for compound geometric sums.

The organization of this paper is as follow. Section 2 deals with some

characterizations of the $\chi^{2}\left(N_{p}\right)$. An algorithm of calculating the probability density function of $\chi^{2}\left(N_{p}\right)$ is presented in this section. In Section 3 the asymptotic behavior of desired normalized sum the asymptotic behaviors of two normalized geometric random sums $p \chi^{2}\left(N_{p}\right)$ and $p^{1 / 2} \sum_{j=1}^{N_{p}}\left[\frac{X_{j}^{2}-1}{\sqrt{2}}\right]$ when $p \searrow 0^{+}$ will be presented in two weak limit theorems for compound geometric sums of squares of independent standard normal random variables. The received results in this

paper are a continuation of the ${ }^{2}$.

\section{CHARACTERIZATIONS OF CHI-SQUARED TYPE RANDOM VARIABLE WITH GEOMETRIC DEGREES OF FREEDOM $\chi^{2}\left(N_{p}\right)$}

For the sake of convenience, we denote by $f_{\chi^{2}\left(N_{p}\right)}(x)$ and $F_{\chi^{2}\left(N_{p}\right)}(x)$ the

probability density function and probability distribution of the chi-square type with geometric random degree of freedom $\chi^{2}\left(N_{p}\right)$, respectively. Based on formula in (1), the following propositions will be stated without proofs as follows:
Proposition 2.1. The density probability function of $\chi^{2}\left(N_{p}\right)$ is given by

$$
\begin{gathered}
f_{\chi^{2}\left(N_{p}\right)}(x)=\sum_{n=1}^{\infty} P\left(N_{p}=n\right) f_{\chi^{2}(n)}(x) \\
=\sum_{n=1}^{\infty} p(1-p)^{n-1} f_{\chi^{2}(n)}(x), \\
\quad x \in(0,+\infty) .
\end{gathered}
$$

According to the formula in (5), the probability density function of the $\chi_{N(p)}^{2}$ should be calculated by following algorithm.

Algorithm 2.1.

1. Define the probability distribution function $f_{\chi^{2}(n)}(x)$ in (1).

2. Compute the probabilities $P\left(N_{p}=n\right)=p(1-$ $p)^{n-1}, n \geq 1$ related to the geometric random variable $N_{p}$ with parameter $p \in(0,1)$.

3. Compute the probability distribution function $f_{\chi^{2}\left(N_{p}\right)}(x)$ with the geometric degrees of freedom $N_{p}$, by the formula (5)

$$
f_{\chi^{2}\left(N_{p}\right)}(x)=\sum_{n=1}^{\infty} p(1-p)^{n-1} f_{\chi^{2}(n)}(x) .
$$

Proposition 2.2. The probability distribution function of $\chi^{2}\left(N_{p}\right)$ is defined as follows

$$
\begin{aligned}
& F_{\chi^{2}\left(N_{p}\right)(x)}=\sum_{n=1}^{\infty} P\left(N_{p}=n\right) P\left(\chi^{2}(n) \leq x\right) \\
& =\sum_{n=1}^{\infty} p(1-p)^{n-1} F_{\chi^{2}(n)}(x), \\
& x \in(0,+\infty), \\
& \text { whereF } \chi^{2(n)}(x)=\int_{0}^{x} f_{\chi^{2}(n)}(x) d x .
\end{aligned}
$$

According to $X_{j} \sim \mathscr{N}_{(0,1)}$, for $j \geq 1$, hence $X_{j}^{2} \sim$ $\chi^{2}(1)$ for $j \geq 1$. Then the numeric characterizations of chi-square type random variable with geometric degree of freedom $\chi^{2}\left(N_{p}\right)$ should be directly calculated as follows:

\section{Proposition 2.3.}

1. Using the Wild's identity for a random sum (see for instance $^{10}$, the mean of $\chi^{2}\left(N_{p}\right)$ should be given in from

$$
\mathbb{E}\left(\chi^{2}\left(N_{p}\right)\right)=\mathbb{E}\left(N_{p}\right) \times \mathbb{E}\left(X_{j}^{2}\right)=p^{-1}
$$

2. The variance of $\chi^{2}\left(N_{p}\right)$ will be computed by

$$
\begin{aligned}
& \operatorname{Var}\left(\chi^{2}\left(N_{p}\right)\right)=\mathbb{E}\left(N_{p}\right) \times \operatorname{Var}\left(X_{j}^{2}\right) \\
& +\left(\mathbb{E}\left(X_{j}^{2}\right)\right)^{2} \times \operatorname{Var}\left(N_{p}\right) \\
& =2 p^{-1}+\left(\frac{1-p}{p^{2}}\right)=\frac{1+p}{p^{2}} .
\end{aligned}
$$




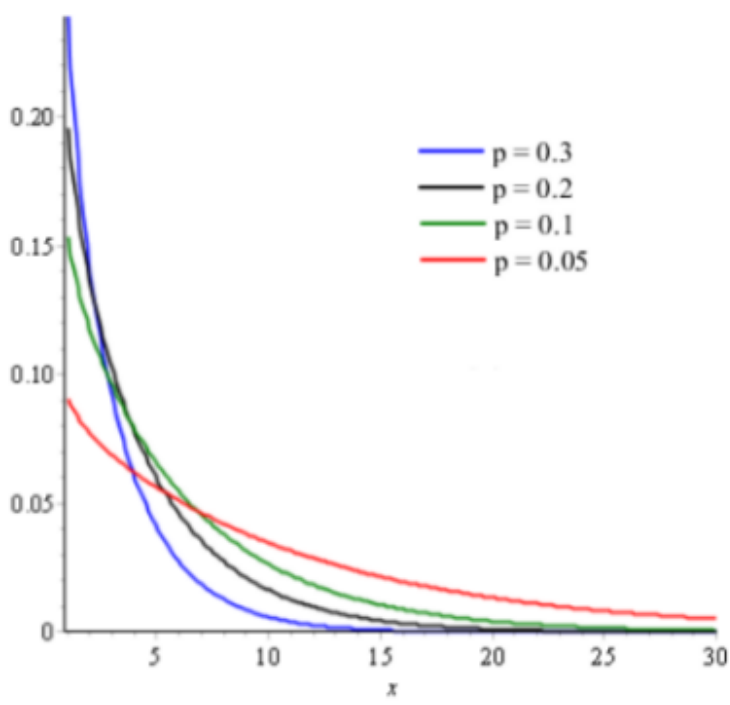

Figure 1: Plot of probability density function $f_{\chi^{2}\left(N_{p}\right)}(x)$ corresponding the geometric parameters $\mathbf{p}, p \in$ $(0,1)$, established by formula (5).

The following figure is showing the behaviors of curves of the probability density functions defined in (5), corresponding various value of parameter $p \in$ $(0,1)$.

Remark 2.1. It is clear that, according to the Figure 1, the curves of the probability density distribution $f_{\chi^{2}\left(N_{p}\right)}(x)$ are decreasing when values of the parameters $p$ tend to zero. This does not allow us to have analogues as asymptotic behaviors of the probability density distribution $f_{\chi^{2}(n)}(x)$ of the chi-square random variable with geometric degrees of freedom $\chi^{2}(n)$ in (1) when $n$ tends to infinity (see ${ }^{1}$ for more details). The essence of this difference will be explained by weak limit theorems for geometric random sums in next section.

\section{ASYMPTOTIC BEHAVIORS OF $\chi^{2}\left(N_{p}\right)$ IN RELATION TO GEOMETRIC RANDOM SUMS}

Here and subsequently, denote by $\mathscr{E}_{\mathrm{m}}$ the exponential distributed random variable with mean $\mathbb{E}\left(\mathscr{E}_{m}\right)=$ $m$, with characteristic function $\varphi_{\varepsilon_{m}}(t)=\frac{1}{1-i t}$, and $\mathscr{D}_{(a)}$ stands for the random variable degenerated at point $a \in(-\infty,+\infty)$, i.e. $P\left(\mathscr{D}_{(a)}=a\right)=$ 1 and $P\left(\mathscr{D}_{(a)} \neq a\right)=0$.

The following theorems will demonstrate the asymptotic behaviors of two

normalized geometric random sums $p \chi^{2}\left(N_{p}\right)$ and $p^{1 / 2} \sum_{j=1}^{N_{p}}\left[\frac{X_{j}^{2}-1}{\sqrt{2}}\right]$ when $p \searrow 0^{+}$.
The received results will show the difference between of limiting distributions of normalized geometric random sums and determined sums in terms of assertions (3) and (4).

Before stating the main results of this section we first provide some propositions as follows.

Proposition 3.1. Let $\mathscr{E}_{m}$ be an exponential distributed random variable with mean $m$. Then,

$$
\varepsilon_{m} \stackrel{D}{=} p \sum_{j=1}^{N_{p}} \varepsilon_{m}^{(j)}
$$

where $\mathscr{E}_{m}^{(j)}$ are i.i.d random variables having exponential distribution with mean $m$, and independent of $N_{p}$ for $p \in(0,1)$. Here and from now on the notation $\stackrel{D}{=}$ stands the identity in distribution.

Proof. According to Theorems 9.1 and 9.2 in ${ }^{10}$ (page 193-194), the characteristic function of $p \sum_{j=1}^{N_{p}} \varepsilon_{m}^{(j)}$ will be defined as follows

$$
\begin{aligned}
& \varphi_{p \Sigma_{j=1}^{N_{p}} \varepsilon_{m}^{(j)}}(t)=h_{N_{p}}\left(\varphi_{\varepsilon_{m}^{(j)}}(p t)\right)= \\
& \frac{p \varphi_{\varepsilon_{m}}^{(j)}(p t)}{1-(1-p) \varphi_{\varepsilon_{m}^{(j)}}(p t)}=\frac{p}{\varphi_{\varepsilon_{m}^{-1}}^{-1}(p t)-1+p} \\
& =\frac{p m}{m-i t-m+p m}= \\
& \frac{m}{m-i t}=\varphi_{\varepsilon_{m}}(t) \text { for } t \in(-\infty,+\infty),
\end{aligned}
$$

where $h_{N_{p}}(t)=\mathbb{E}\left(t^{N_{p}}\right)$ denotes the probability generating function of $N_{p}$. The Eq. (10) finishes the proof. 
Theorem 3.1. Let $\left\{X_{n} n \geq 1\right\}$ be a sequence of independent, standard normal distributed random variables $X_{n} \sim \mathscr{N}_{(0,1)}$ for $n \geq 1$. Let $N_{p}$ be a geometric distributed random variable with parameter $p, p \in(0,1)$. Assume that the random variables $X_{1}, X_{2}, \ldots$ and $N_{p}$ are independent. Then,

$$
p \chi^{2}\left(N_{p}\right)=p \sum_{j=1}^{N_{p}} X_{j}^{2} \stackrel{D}{\rightarrow} \varepsilon_{1} \text { as } p \searrow 0^{+},
$$

where $\varepsilon_{1} \sim \operatorname{Exp}(1)$ is an exponential distributed random variable with mean 1 and

$$
\mathbb{P}\left(\varepsilon_{1} \leq x\right)=1-e^{-x} \text { for } x \geq 0
$$

Proof. Let us denote by $h_{N_{p}}(t):=$ $\mathbb{E}\left(t^{N_{p}}\right)$ and $\varphi_{X_{n}^{2}}(t):=\mathbb{E}\left(e^{i t X_{n}^{2}}\right)$ the probability generating function of $N_{p}$ and the characteristic function of a random variable $X_{n}$, respectively. Then, direct computation shows that

$$
h_{N_{p}}(t)=\frac{p t}{1-(1-p) t},
$$

for $|t|<\frac{1}{1-p}, p \in(0,1)$,

and

$$
\varphi_{X_{n}^{2}}(t)=(1-2 i t)^{-1 / 2}
$$

for $-\infty<t<+\infty, n \geq 1$.

In view of theorems 9.1 and 9.2 in $^{10}$ (page 193-194), the characteristic function of the $p \chi^{2}\left(N_{p}\right)$ is given by

$$
\begin{array}{r}
\varphi_{p \chi^{2}\left(N_{p}\right)}(t)=h_{N_{p}}\left(\varphi_{X_{n}^{2}}(p t)\right)=\frac{p \varphi_{X_{n}^{2}}(p t)}{1-(1-p) \varphi_{X_{n}^{2}}(p t)}= \\
\frac{p}{\sqrt{1-2 i p t}-(1-p)}=\frac{p[\sqrt{1-2 i p t}+(1-p)]}{1-2 i p t-(1-p)^{2}}= \\
\frac{\sqrt{1-2 i p t}+1-p}{2-2 i t-p}
\end{array}
$$

Letting $p \rightarrow 0^{+}$, we can assert that

$\varphi_{p \chi^{2}\left(N_{p}\right)}(t) \rightarrow(1-i t)^{-1}=\varphi_{\varepsilon_{1}}(t)$ for all $t \in(-\infty,+\infty)$.

In view of the continuity theorem for characteristic function (see ${ }^{10}$ for more details), the proof is finished. Remarks 3.1. Theorem 3.1 is an analog of the Rényi's result (1957) on asymptotic behavior of geometric random sum of independent, identically positive-valued random variables with positive mean (see ${ }^{6}$ and ${ }^{5}$ for more details).

It makes sense to consider that the assertion in (4) will not be valid if the non-random number $n$ (being degrees of freedom) is replaced by a geometric random variable $N_{p}, p \in(0,1)$. The next thereom 3.2 will present the asymptotic behavior of a normalized geometric sum $p^{1 / 2} \sum_{j=1}^{N_{p}}\left[\frac{X_{j}^{2}-1}{\sqrt{2}}\right]$, when $p \searrow 0^{+}$.
Proposition 3.2. The Laplace distributed random variable $\mathscr{L}_{(0,1)}$ with zero location parameter and unit scale parameter should be presented in following form

$$
\mathscr{L}_{(0,1)} \stackrel{D}{=} p^{1 / 2} \sum_{j=1}^{N_{p}} \mathscr{L}_{(0,1)}^{(j)},
$$

where $\mathscr{L}_{(0,1)}^{(j)}, j \geq 1$ are i.i.d. Laplace distributed random variables with parameters 0 and 1 , independent of $\mathrm{N}_{p}$ forp $\in(0,1)$

Proof. We shall begin with showing that the characteristic function of $\mathscr{L}_{(0,1)}^{(j)}$ at point $p^{1 / 2} t$ is given by

$$
\varphi_{\mathscr{L}_{(0,1)}}^{(j)}\left(p^{\frac{1}{2}} t\right)=\left(1+\frac{1}{2} p t^{2}\right)^{-1}
$$

Then

$$
\begin{gathered}
\varphi_{p^{\frac{1}{2}} \sum_{j=1}^{N_{p}} \mathscr{L}_{(0,1)}^{(j)}}(t)=h_{N_{p}}\left(f_{\mathscr{L}_{(0,1)}^{(j)}}\left(p^{\frac{1}{2}} t\right)\right)= \\
\frac{p \varphi_{\mathscr{L}_{(0,1)}}\left(p^{\frac{1}{2}} t\right)}{1-(1-p) f_{\mathscr{L}_{(0,1)}^{(j)}}^{\left(j-p^{\frac{1}{2}} t\right)}}=\left(1+\frac{1}{2} t^{2}\right)^{-1} \\
=\varphi_{\mathscr{L}_{(0,1)}}(t) \text { for } t \in(-\infty,+\infty) .
\end{gathered}
$$

According to the continuity theorem for characteristic function ( ${ }^{10}$, Theorem 9.1, page 238), the proof is finished.

Theorem 3.2. Let the assumptions of the Theorem 3.1 hold. Then

$$
p^{\frac{1}{2}} \sum_{j=1}^{N_{p}}\left[\frac{X_{j}^{2}-1}{\sqrt{2}}\right] \stackrel{D}{\rightarrow} \mathscr{L}_{(0,1)} \text { as } p \searrow 0^{+} .
$$

where $\mathscr{L}_{(0,1)}$ stands for the Laplace distributed random variable with parameters 0 and 1 , having characteristic function in form $\varphi_{\mathscr{L}_{(0,1)}}(t)=\left(1+\frac{1}{2} t^{2}\right)^{-1}$.

Proof. Without loss of generality we may assume that

$$
\frac{x_{j}^{2}-1}{\sqrt{2}}=W_{j}^{2} \text { for } j \geq 1
$$

Then, for $j \geq 1$, we have $\mathbb{E}\left(W_{j}^{2}\right)=0$ and $\mathbb{D}\left(W_{j}^{2}\right)=$ 1. Using Maclaurin series for characteristic function $\varphi_{W_{j}^{2}}\left(p^{\frac{1}{2}} t\right)$, we have

$$
\varphi_{W_{j}^{2}}\left(p^{\frac{1}{2}} t\right)=1-\frac{1}{2} p t^{2}+o\left(\frac{p t^{2}}{2}\right)
$$


It can be verified that

$$
\begin{aligned}
& \varphi_{p}^{\frac{1}{2} \sum_{j=1}^{N_{p}} W_{j}^{2}} \quad(t)=\mathbb{E}\left(e^{i t p \frac{1}{2} \sum_{j=1}^{N_{p}} W_{j}^{2}}\right)= \\
& p \varphi_{W_{j}^{2}}\left(p^{\overline{2}} t\right) \\
& \overline{1-(1-p) \varphi_{W_{j}^{2}}\left(p^{\frac{1}{2}} t\right)} \\
& =\frac{p}{\varphi_{W_{j}}^{-1}\left(p^{\frac{1}{2}} t\right)-1+p}= \\
& \frac{p}{\left[1-\frac{1}{2} p t^{2}+o\left(\frac{p t^{2}}{2}\right)\right]^{-1}-1+p} \text {. }
\end{aligned}
$$

In view of the continuity theorem for characteristic function (see ${ }^{10}$ for more details), the proof is complete.

\section{COMPETING INTERESTS}

None of the authors reported any conflict interest related to this study.

\section{REFERENCES}

1. Hogg RV, McKean J, Craig AT. Introduction to Mathematical Statistics. Seventh Edition, Pearson; 2013. .

2. Hung $T L$, Thanh TT, Vu BQ. Some results related to distribution functions of chi-square type with random degrees of freedom. Bulletin of the Korean Mathematical Society. 2008;45(3):509522.

3. Asmusen S. Applied Probability and Queues. Springer; 2003.

4. Asmusen S. Riun Probabilities. World Scientific; 2010.

5. Kruglov VM, Korolev VY. Limit Theorems for Random Sums, Moskov.Gos. Univ., Moscow, 1990.

6. Kalashnikov V. Geometric sums: bounds for rare events with applications. Risk analysis, reliability, queuing. Mathematics and its Applications, 413. Dordrecht: Kluwer Academic Publishers Group; 1997. .

7. Gnedenko BV, Korolev VY. Random Summations: Limit Theorems and Applications. New York: CRC Press; 1996. .

8. Bon JL. Geometric Sums in Reliability Evaluation of Regenerative Systems. Information Processes. 2002;2(2):161-163.

9. Grandell J. Risk Theory and Geometric Sums. Information Processes. 2002;2(2):180-181.

10. Gut A. Probability: a graduate course. Springer Texts in Statistics. New York: Springer; 2005. 